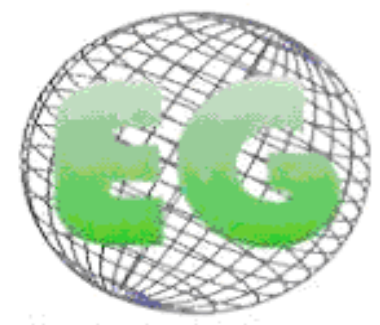

ISSN 1695-6141

N 27
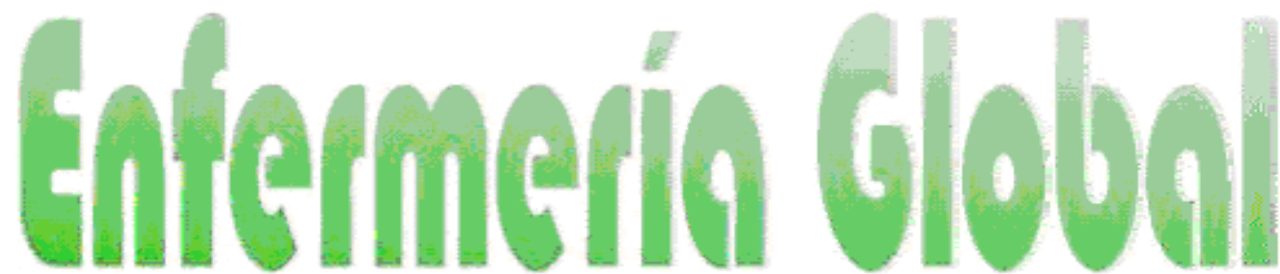

Revista electrónica trimestral de Enfermería

Julio 2012

www.um.es/egloball

\title{
“¿Quién soy yo?” con la palabra, enfermeros supervisores de un hospital privado de Belo Horizonte “
}

Quem sou eu"? com a palavra, enfermeiros-supervisores de um hospital privado de Belo Horizonte

*Ferreira Roquete, F., **Menezes Brito., MJ., ***Lopes Melo, MCO., "***Marques, AL., ${ }^{* * * * *}$ Mendes, LN.

*Profesora Asistente. ENA, Escuela de Enfermería de la UFMG. Miembro del Núcleo de Investigación Administración en Enfermería (NUPAE). E-mail: fatimaroquete@gmail.com **Profesora Adjunta, ENA, Escuela de Enfermería, UFMG. Vice-Líder del Núcleo de Investigación Administración en Enfermería (NUPAE). ${ }^{* * *}$ Directora General de la Facultad Novos Horizontes, Vice-Diretora de Pos-Graduación de la Facultad Novos Horizontes. ${ }^{* * *}$ Profesor Adjunto. CEPEAD, Departamento de Ciencias Administrativas, FACE UFMG. ${ }^{* * * * *}$ Coordinadora de Enfermería del Hospital Domiciliar Ltda. Brasil.

Palabras clave: Ego; Supervisión de Enfermería; Gestión en Salud; Formación de Recursos Humanos. Palavras-chave: Ego; Supervisão de Enfermagem; Gestão em Saúde; Formação de Recursos Humanos. Keywords: Ego; Nursing Supervision; Health Management; Human Resources Formation

\section{RESUMEN}

En este artículo se pretende comprender cómo los enfermeros-supervisores de un gran hospital privado ubicado en Belo Horizonte construyen su identidad. Para ello, se realizó un estudio de caso descriptivo y analítico que incluyó a nueve enfermeros-supervisores. La recolección de datos se llevó a cabo a través de entrevistas con guión semiestructurado y el análisis mediante la técnica de análisis de contenido, que abarcó las siguientes unidades de análisis: la percepción de los enfermeros-supervisores en cuanto al nuevo método de gestión implantado en el hospital; el trabajo cotidiano de los enfermeros-supervisores; la formación en enfermería y la práctica profesional; los requisitos de un enfermero ideal para el puesto de gestión, y las expectativas de carrera de los enfermeros-supervisores. Los resultados mostraron que el nuevo modo de gestión adoptado por el hospital se caracterizó como un espacio significativo de reconfiguración de identidad de los enfermeros-supervisores, en particular, por poner de relieve el desnivel entre la formación académica y la práctica cotidiana del trabajo.

\section{RESUMO}

O presente artigo teve como objetivo compreender como enfermeiros-supervisores de um hospital privado de grande porte situado em Belo Horizonte constroem sua identidade. Para tanto, foi realizado um estudo de caso descritivo-analítico, do qual participaram nove enfermeiros supervisores. A coleta de dados foi realizada por meio de entrevista com roteiro semi-estruturado e a análise utilizando-se a técnica de análise de conteúdo, que 
contemplou as unidades de análise: a percepção dos enfermeiros-supervisores quanto ao novo modo de gestão implantado no hospital; o cotidiano de trabalho dos enfermeiros-supervisores; a formação em enfermagem e a prática profissional; os requisitos de um enfermeiro ideal para a função gerencial; e expectativas de carreira dos enfermeiros-supervisores. Os resultados mostraram que o novo modo de gestão adotado pelo hospital caracterizou-se como espaço significativo de reconfiguração identitária dos enfermeirossupervisores, particularmente, por marcar o descompasso entre a formação acadêmica e as práticas no cotidiano do trabalho.

\section{ABSTRACT}

This paper aims to understand how supervisor-nurses of a big private hospital in Belo Horizonte build their professional identity. In order to do that, a descriptive analytical case study was carried out in which nine supervisor nurses were included. Data were collected through semi-structured interviews and the analysis by means of the content analysis technique. The analysis focused on: the supervisor nurses' perception concerning the new management procedure used at the hospital; supervisor-nurses' routine at work; nursing education and professional practice; qualifications of an ideal nurse for the management position; and supervisor-nurses' carrier expectations. The results showed that the new management strategy used by the hospital was considered a significant space for the supervisor-nurses' identity reconfiguration, especially because it demonstrates a disharmony between academic instruction and daily practice at work.

\section{INTRODUCCIÓN}

En el actual contexto de trabajo de enfermería en los hospitales privados, se observa la presencia de dificultades en cuanto a la claridad de las atribuciones ejercidas por el enfermero, tanto en lo que respecta a su actuación en el equipo de enfermería como también en cuanto a miembro de un equipo interdisciplinar. En lo que respecta a las verdaderas atribuciones del enfermero, Brito et al. ${ }^{1}$ evidencian que la participación de este profesional en actividades gerenciales y asistenciales ha representado, para muchos, una situación generadora de conflictos dado que, en diversas situaciones asumen un vasto abanico de actividades cuya priorización y conciliación se tornan prácticamente inviables.

A pesar de que la formación académica proporciona conocimientos concernientes al proceso gerencial y asistencial, el enfermero ha vivido conflictos e incertidumbres, conforme se ha constatado. $^{2}$ En el actual contexto de hospitales privados de Belo Horizonte, el enfermero viene incorporando otras demandas a la actividad gerencial, que sumadas a aquellas intrínsecas y privativas a su profesión, propician la presencia de dicotomía entre lo que se espera de él como profesional, en la visión de los teóricos de enfermería, y lo que es practicado en el cotidiano del trabajo. ${ }^{1}$

Es conocido que "la entrada a una especialidad disciplinaria [...] constituye un acto significativo de la identidad". 3:146 Para Dubar ${ }^{4}$, la identidad humana es

el resultado a un mismo tiempo estable y provisorio, individual y colectivo, subjetivo y objetivo, biográfico y estructural, de los diversos procesos de socialización que, conjuntamente, construyen los individuos y definen las instituciones. ${ }^{4: 136}$

De esta forma, la actividad profesional, el trabajo, es una dimensión primordial en el proceso de constitución de identidad. Cabe recordar que es "en la confrontación con el mercado de trabajo donde, ciertamente, se ubica la implicación de identidad más importante de los individuos" 3:146, y que condiciones históricas particulares, entre ellas, el "rápido proceso de modernización tecnológica y de cambios organizacionales en las empresas"3:149 predisponen cuestionamientos de identidades anteriormente adquiridas. De esa confrontación

dependerán las modalidades de construcción de una identidad profesional básica que constituya no solo una identidad en el trabajo, sino también, y sobre todo, una proyección de 
sí en el futuro, la anticipación de una trayectoria de empleo y la elaboración de una lógica de [...] formación. ${ }^{4: 149}$

En un estudio sobre la formación del enfermero, Gabrielli ${ }^{5}$ afirma que el proceso formativo todavía parece estar desvinculado de la práctica profesional, llevando a una disparidad entre lo que aprende y lo que vive. Con la expansión de las carreras de graduación en Enfermería y la reformulación de sus currículos, los enfermeros se encuentran, muchas veces, distantes de la realidad de los contextos organizacionales. Se constata que los currículos privilegian el gerenciamiento y la administración tanto de la fuerza de trabajo como de los recursos físicos y materiales y de información, de la misma forma que indican la necesidad de que los profesionales estén preparados para ser emprendedores, gestores, empleadores o líderes en el equipo de salud. ${ }^{6}$ En ese contexto, surgen las políticas de educación dirigidas a la formación basada en competencias para adecuar los sistemas educacionales hacia un nuevo concepto para la calificación profesional, buscando reducir la divergencia entre formación académica y práctica profesional. ${ }^{7}$

Los enfermeros han vivido una nueva realidad en el contexto del trabajo en hospitales privados de Minas Gerais. ${ }^{1,8,9}$. Dicho contexto viene sufriendo continuas modificaciones, particularmente, a partir de la década de los 90, por influencia de diversos factores. Entre ellos, destaca el hecho de que el sector privado de salud se ha vuelto más competitivo, como resultado de la reglamentación estatal de los planes y seguros privados - segmento suplementario de salud - y del ingreso de capitales internacionales, cuando las organizaciones privadas de salud recibieron un significativo impulso para cambiar las formas de gestión. ${ }^{10}$ Dichos cambios, según Pompeu ${ }^{11}$, objetivaron la reducción de los costos, la descentralización de las acciones y la orientación hacia el mercado. Según el mismo autor, las transformaciones significaron el establecimiento de indicadores y metas; los prestadores tuvieron medios y autonomía para alcanzarlas; se incentivó la competencia entre los servicios, como también, la libertad de que el usuario elija el mejor servicio; autonomía y calidad de los servicios se convirtieron en lemas.

Teniendo en cuenta lo planteado, el objetivo general de este trabajo fue analizar la configuración de identidad de enfermeros supervisores de un hospital privado de gran porte ubicado en Belo Horizonte. Específicamente, se buscó: a) discutir la influencia de la formación académica en enfermería en la actuación del enfermero supervisor; b) conocer las percepciones de los enfermeros supervisores en cuanto a su cotidiano de trabajo; c) comprender cómo se construye la identidad de los enfermeros supervisores en el contexto de nuevas tecnologías gerenciales.

\section{IDENTIDAD}

La identidad ha sido tema de interés de los estudios de hechos sociales en el contexto contemporáneo, pues tanto la identidad personal como la social contribuyen a la comprensión de las prácticas individuales y colectivas en la actualidad. ${ }^{12}$ Los estudios de la identidad se concentran en los campos del conocimiento de la Psicología, de la Sociología, del Psicoanálisis y Antropología, y más recientemente, de la Teoría Organizacional ${ }^{13}$, siendo adoptados enfoques diferentes. ${ }^{3,4,14-21}$. El análisis de la identidad depende, entonces, del enfoque que se vaya a utilizar. ${ }^{1,22}$

Tal situación favorece que ambigüedades e imprecisiones permeen la utilización del término identidad, muchas veces utilizado con sentido de identificación. Por un lado, identidad "se refiere a la autodefinición del sujeto, en la interacción entre el yo y la sociedad, entre lo interior y lo exterior, mientras que la identificación se refiere a la obtención por parte del 
sujeto de las cualidades del otro, en el sentido de elevar su autoestima. Además, el término identidad busca responder a la cuestión “¿Quién soy yo?”, mientras que la identificación se refiere a la obtención por parte del sujeto de las cualidades del otro, en el sentido de elevar su autoestima. ${ }^{13: 2}$

Coley, Mead y Goffman fueron los primeros investigadores que estudiaron la identidad, en cuanto fenómeno social, bajo una perspectiva social y contextual ${ }^{23}$. Cooley $^{24}$ considera que el self (identidad) es establecido en nivel social, siendo producto de una relación orgánica entre sociedad y el individuo. En la perspectiva de $\mathrm{Mead}^{25}$, el self (identidad social) se

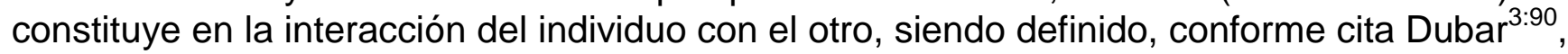
como "producto de la interacción y comunicación entre los individuos, en donde el sujeto toma conciencia de su identidad y adopta los puntos de vista dominantes en el grupo social en el que se encuadra y que comparte".

Por otro lado, Goffman ${ }^{26}$ destaca la expresividad del individuo (capacidad de dar impresión) como actividad significativa en la constitución del yo (identidad), así como demuestra la influencia recíproca de los individuos sobre las acciones entre unos y otros, en cuanto presencia física inmediata. El mismo autor subraya que en esa interacción social el individuo representa roles, preestableciendo un patrón de acción que será desarrollado durante la representación. Goffman ${ }^{27}$ amplió el concepto de representación, presentando categorías de identidad: identidad social virtual (identidad para el otro), identidad social real (identidad para sí) e identidad personal, relacionada, según Fernandes (2008, p. 29) "a las marcas positivas y a la combinación de los ítems de la historia de vida incorporados por el individuo, presuponiendo su unicidad y diferenciación".

De esta forma, la identidad va siendo construida, a lo largo de la vida, intrínsecamente relacionada al contexto social. ${ }^{16}$ Sin embargo, el individuo no construye su identidad de una vez; él la construye en la infancia y debe reconstruirla siempre. ${ }^{3,4}$ La identidad, siempre en formación, contempla la articulación de lo que es interno al individuo con las formas conforme él imagina ser visto por otros. ${ }^{19}$ Ese otro se remite al Psicoanálisis, en que Freud ${ }^{14}$ identifica el significado crucial del primer otro - la madre - para la constitución del sujeto. El círculo de significativos otros va a ampliarse, pudiendo ir "desde la persona materna hasta la humanidad" 15:21. La internalización del mundo exterior por parte del sujeto y la externalización de su mundo interior se dan por medio de la acción en el mundo social. ${ }^{19}$

A la articulación entre las dos transacciones "una transacción interna al individuo y una externa establecida entre el individuo y las instituciones con las cuales interactúa" 3:102 o sea, con el mundo externo, Dubar ${ }^{3}$ denomina identidad social. Esta está marcada por una dualidad y comprende dos procesos: el relacional (para el otro) y el biográfico (para sí). Según él, la noción de identidad puede ser incluida en una perspectiva sociológica "si restituimos esta relación identidad para sí / identidad para el otro en el interior del proceso común que la torna posible y que constituye el proceso de socialización" 3:105.

La socialización es la "amplia y consistente introducción de un individuo en el mundo objetivo de una sociedad o de un sector de ella"28:175. Los autores distinguen dos formas de socialización: la primaria y la secundaria. La socialización primaria es aquella que el individuo vive en la infancia, y en virtud de la cual se vuelve miembro de la sociedad. La socialización secundaria se refiere a cualquier proceso subsiguiente que introduce un individuo, ya socializado, en nuevos sectores del mundo objetivo de su sociedad. Dubar ${ }^{4}$ entiende que, entre esos sectores, se destaca el mundo laboral y su significado en la construcción de la identidad. Destaca que la identidad profesional no puede resumirse a la identidad en el trabajo, en la medida en que contempla también la proyección de sí en el 
futuro. Además de la elección de un "oficio o diploma", se trata de la construcción personal de una trayectoria, en la cual está presente tanto la capacidad de que el individuo realice sus deseos como la imagen que tiene de sí mismo.

Aspectos de la autoimagen de un individuo, que derivan de las categorías sociales que él percibe como perteneciente, constituyen su identidad social en el ámbito organizacional. Las identificaciones en ese contexto son, en gran medida, relacionales y comparativas: ellas definen al individuo como semejantes o diferentes, como "mejores" o "peores" que integrantes de otros grupos. Hay tres componentes del proceso de identificación del sujeto con las diferentes categorías y grupos sociales. El cognitivo, por medio de la autodefinición y autocategorización del sujeto como perteneciente a un grupo; el afectivo, que es retratado por los sentimientos del sujeto con relación al hecho de pertenecer a determinado grupo social, y el valorativo, relacionado al valor asignado al grupo por los grupos externos. ${ }^{17,18}$

El mundo actual está marcado por incertidumbres y cambios continuos. Las realizaciones individuales no se solidifican en poses permanentes, pues los activos se transforman en pasivos, y las capacidades en incapacidades. ${ }^{21}$ Las condiciones de acción y las estrategias de reacción envejecen y se vuelven obsoletas. Tanto la esfera de formación escolar y profesional como las esferas del trabajo y del empleo se muestran marcadas por rupturas. Esos espacios son prioritarios en la configuración de identidad de los individuos. Considerando que la identidad profesional de base no es fija, ni definitiva, factores como la modernización tecnológica y las transformaciones organizacionales van a implicar, muchas veces, en retomar identidades anteriormente construidas. ${ }^{4}$ El contexto de actividad profesional de los enfermeros estudiados pasa por cambios que son propios del ambiente empresarial contemporáneo y se caracteriza por la implantación de nuevas formas de gestión.

\section{NUEVAS FORMAS DE GESTIÓN}

En las últimas décadas, la sociedad viene pasando por innumerables transformaciones influenciadas por el pensamiento empresarial, que valora la economía, las empresas y la gestión. En esa dirección, crecen también demandas por la búsqueda continua de alternativas que respondan a las exigencias de la innovación y de la competitividad de las organizaciones. $^{29}$ Mintzberg $^{30}$ señala características de la gestión en ese contexto, mencionando riesgos tanto para el negocio como para las personas. Él denomina el trabajo de los ejecutivos, de "saga heroica". Esos profesionales pasan a vivir una intensa presión por productividad y reducción de costos, responsabilización sobre el desempeño de toda la empresa, empoderamiento, estrategias diversificadas de motivación, en fin, todo lo que es necesario para "elevar el desempeño mensurable a corto plazo, sin importar cómo" 30:19.

Otros trabajadores, entre los cuales, miembros de la mediana administración, también son expuestos a la presión por productividad, por eficiencia, al despido y a las enfermedades como el estrés crónico. ${ }^{30}$ Bauman ${ }^{21: 9}$ resalta que "la vida en una sociedad líquido moderna no puede quedar parada". La cuestión central es una intensa presión por "modernizarse (entiéndase: ir adelante despojándose a cada día de los atributos que excedieron la fecha de vencimiento y desmantelamiento, repeliendo las identidades que actualmente están siendo montadas y asumidas) o perecer" ${ }^{\prime 2}$.

Modificaciones significativas de la gestión de las organizaciones de salud privadas, en general, y de los hospitales, en particular, sucedieron de forma más expresiva a partir de la década del 90. Los cambios, tanto en los procesos de producción como en la organización del trabajo en sí, consecuencia de aspectos más amplios como la internacionalización de los 
mercados, la reestructuración productiva, los avances en tecnología y las cuestiones referentes a la cultura, han exigido de las organizaciones un reposicionamiento ante el modelo tradicional. Este, caracterizado por principios como la jerarquía, la especialización por funciones, las unidades de mando, los períodos operacionales anuales, entre otros, tanto desde el punto de vista de la gestión como del negocio, se tornó insuficiente para atender las necesidades de las organizaciones. ${ }^{1,8,9,31,32}$ El modelo tradicional se fundamenta en nuevos principios, tales como la asignación de recursos en tiempo real, la comunicación punto a punto, la organización del trabajo en grupos y proyectos, la evaluación de desempeño por resultados y las fronteras orgánicas. ${ }^{33}$ Sin embargo, Castro et al. ${ }^{34}$ resaltan que hay un desafío latente

\begin{abstract}
que consiste en el esfuerzo de transformar empleados de tareas en profesionales de proceso; en el repensar de los roles de los gestores y de los empleados en esa nueva organización; en el reinvento de los sistemas de gestión; en hacer que el aprendizaje sea parte del día a día de los negocios de la empresa, así como moldear una nueva cultura que brinde soporte a la nueva manera de trabajar. ${ }^{34: 107}$
\end{abstract}

Teniendo en cuenta la especificidad del sector salud, cabe recordar que los desafíos asignados a los hospitales privados fueron reforzados por la necesidad de integración a las redes de atención preconizadas por el Sistema Único de Salud (SUS), significando un impulso adicional para modificaciones conceptuales y operacionales en las formas de gestión. La implantación de un nuevo modelo de organización de servicios "por equipos" significó la necesidad de reemplazar la lógica vigente de organización de servicios "por profesiones" ${ }^{32}$, entre otras modificaciones resultantes de la acreditación. Esta se encuentra definida como

[...] un sistema de evaluación y certificación de calidad de servicios de salud, voluntario, periódico y reservado, siendo una acción coordinada por una organización o agencia no gubernamental encargada dell desarrollo y actualización de su metodología. En sus principios, se presenta como una acción de carácter educativo, enfocada a la mejora continua, sin propósitos de fiscalización o control oficial. ${ }^{35}$

Los criterios de evaluación están relacionados con las siguientes categorías: los liderazgos y la administración, en lo que respecta a la dirección, la administración en sí y a la gestión de calidad; los servicios profesionales y organización de la asistencia, ítem que afecta directamente el trabajo de enfermería; servicio de atención al paciente/cliente; servicios de apoyo al diagnóstico; servicios de apoyo técnico y abastecimiento; servicios de apoyo administrativo e infraestructura; y enseñanza e investigación. ${ }^{36}$

Es en el espacio de un hospital privado de gran porte, ubicado en Belo Horizonte, Minas Gerais, inserto en ese contexto, donde enfermeros supervisores tienen la palabra y responden a la cuestión “¿Quién soy yo?”

\title{
RECORRIDO METODOLÓGICO
}

Para responder a la cuestión propuesta, se adoptó el abordaje cualitativo, de carácter descriptivo analítico. Se parte del principio de que hay una relación dinámica entre el mundo real y el sujeto y que el conocimiento no se reduce a un inventario de datos aislados, conectados a una teoría explicativa, es decir, "el sujeto observador es parte integrante del proceso de conocimiento e interpreta los fenómenos, asignándoles un significado" ${ }^{37: 79}$.

El estudio descriptivo presupone la existencia de algún conocimiento preliminar sobre el asunto a ser estudiado y tiene como objetivo la descripción de las características del objeto 
elegido, exponiendo características de determinada población o fenómeno, buscando comprenderlos como un todo. ${ }^{38} \mathrm{El}$ estudio de caso fue elegido por el hecho de contribuir a la comprensión de fenómenos sociales complejos y el contexto relativo a esos fenómenos ${ }^{39}$, siendo la manera apropiada de aprender aspectos de la identidad del enfermero supervisor que actúa en el contexto hospitalario privado.

El escenario del estudio fue un hospital privado de gran porte ubicado en Belo Horizonte, Minas Gerais, que, para preservarlo será denominado ficticiamente HMAl en este estudio. Los sujetos fueron diecisiete enfermeros, pertenecientes al cuadro de personal del hospital, asignados a las unidades de internación, Unidad de Terapia Intensiva (UTI) y bloque quirúrgico. Como criterio para participar en la investigación, el profesional debería estar ejerciendo la función de supervisor del equipo de enfermería, independientemente del tiempo de ejercicio.

La recolección de datos ocurrió tras la aprobación del Comité de Ética en Investigaciones de la Universidad Federal de Minas Gerais (UFMG) y del hospital. En cumplimiento a lo dispuesto en el art. 4º de la Resolución 196/96 del Consejo Nacional de Salud, el término de consentimiento libre y aclarado fue firmado por los sujetos de la investigación. Los datos fueron recogidos por medio de entrevista con guía semiestructurada, instrumento que hizo posible "orientar una conversación con finalidad [...] ser el facilitador de apertura, de ampliación y de profundización de la comunicación”, y participación más activa de los investigadores ${ }^{40: 99}$.

El análisis de los datos primarios fue realizado por medio de la técnica de análisis de contenido ${ }^{41}$ trás la íntegra transcripción de las grabaciones, lectura minuciosa de las entrevistas e identificación de los temas relevantes que surgieron en los discursos de los entrevistados. Enseguida, los datos extraídos fueron relacionados y clasificados de acuerdo con categorías temáticas preestablecidas: percepción de los enfermeros supervisores en cuanto a la nueva forma de gestión del HMAl y su cotidiano de trabajo; la formación en enfermería y la práctica profesional; requisitos de un enfermero ideal para la función gerencial; y expectativas de carrera de los enfermeros supervisores. Esos datos se refieren a la percepción de enfermeros sobre su formación y actuación profesional, elementos de su configuración de identidad en el contexto de nuevos modelos de gestión implantados en el hospital privado donde actúan. Los datos secundarios, documentos e informes del hospital, fueron analizados por medio de la técnica de análisis documental. ${ }^{41}$

\section{“¿QUIÉN SOY YO”? CON LA PALABRA, LOS ENFERMEROS SUPERVISORES}

Esta sección se destina a la presentación de los resultados de la investigación. Teniendo en cuenta los objetivos propuestos, el análisis de los datos recogidos, precedido por una caracterización del escenario de estudio, está dividido conforme las categorías de análisis, precedidas por la caracterización del hospital escenario de estudio.

EI HMAI forma parte de la red de servicios de salud de la ciudad de Belo Horizonte, habiendo sido fundado en la década de los 50 . En el momento de la recolección de datos, contaba con más de 300 camas distribuidas en habitaciones y enfermerías, UTI adulto, UTI cardiovascular, UTI pediátrico, salas de cirugía y salas de videoendoscopia, además de una Guardia General en funcionamiento y la previsión de la instalación de una Guardia Pediátrica. Actuaba en el HMAl un conjunto de más de 1500 colaboradores del área técnica y administrativa, alrededor de 400 médicos y 100 enfermeros. Se prestaba atención en 24 especialidades médicas, varias de ellas con admisión en pediatría. Los servicios eran 
destinados a clientes particulares, a clientes de más de 60 planes de salud y a clientes del SUS, tanto a nivel ambulatorio como hospitalario.

El HMAl está acreditado por el Ministerio de Salud para atenciones de mediana y alta complejidad, y fue acreditado en nivel pleno por la ONA. Dicha certificación implicó la necesidad de realización de una serie de cambios gerenciales, estructurales y asistenciales. En lo que respecta a la enfermería, interés particular del presente estudio, el hospital tiene como objetivo el perfeccionamiento continuo de sus servicios, a la luz del binomio enseñanza aprendizaje, pautado en la utilización de conocimientos técnico-científicos. Algunas actividades identificadas en el proceso de reorganización de esa área fueron: diagnóstico situacional administrativo y asistencial de todas las unidades de internación; elaboración de la cadena cliente/proveedor; reestructuraciones del proyecto de educación continua; redimensionamiento del equipo; elaboración de protocolos; definición de indicadores asistenciales; contratación exclusiva de técnicos de enfermería y enfermeros; implantación del proceso de enfermería en las unidades de ingreso, por medio de nuevos formularios de examen físico y evolución de los profesionales involucrados; e implementación de la Comisión de Ética del sector (Datos de investigación).

Frente a los cambios identificados en el HMAI, se buscó conocer, por medio de categorías preestablecidas, la percepción de los enfermeros supervisores entrevistados en cuanto a la nueva forma de gestión del hospital, teniendo en cuenta el hecho de que elementos como la modernización tecnológica y las transformaciones organizacionales influyen en la configuración de identidad de los profesionales.

\section{Percepción de los enfermeros supervisores en cuanto a la nueva forma de gestión del HMAI}

El escenario de estudio está inserto en un mundo caracterizado por cambios rápidos y continuos, pudiendo ser calificado como un "mundo líquido moderno", en el cual lo efímero es la palabra orden. ${ }^{21}$ A pesar de que HMAl se muestra como ambiente de cambios continuos, en la percepción de los entrevistados una importante ruptura marcó su trayectoria. La forma de gestión tradicional, caracterizada por principios como jerarquía y unidades de mando, entre otros ${ }^{33}$, fue siendo reemplazado por un nuevo modelo que pudiera sostener la adaptación al contexto y a la supervivencia del HMAI, conforme relatos.

\footnotetext{
El hospital pasó por muchos cambios. Tenía un directorio muy cerrado. Un grupo de médicos controlaba el hospital y listo. Precisó que el [nombre del hospital] cumpla 50 años para que notara que no daba para continuar como estaba. $O$ dividía las responsabilidades, valoraba a las personas que tenían aquí dentro o entonces no iba adelante. Hoy tenemos esa división de gerencias, entonces distribuyó las responsabilidades. Claro que hay un superintendente, que lo que él dice es así, casi como una ley. (E01).
}

A pesar de los cambios implementados, se observan características del modelo centrado en las decisiones médicas. Se verifica que hay un camino recorrido hacia la gestión participativa, observando una disonancia entre lo real y lo prescripto, conforme menciona Chanlat $^{29}$.

Los hospitales están cambiando mucho. La visión del hospital es otra. Uno está más volcado hacia la visión de la empresa más que para un hospital. Hoy nos preocupamos mucho más, por ejemplo, con enfoque en el cliente. Tenemos que ser mejor, ah, tiene que ser el mayor hospital (E05). 
En el caso de los hospitales, burocracias profesionales ${ }^{42}$ la tendencia es a que los cambios ocurran de forma gradual, atendiendo a los diversos desafíos surgidos cuando se propone modificar el modelo tradicional médico centrado. ${ }^{31}$ La estrategia de creación de unidades sectoriales, descentralización y la autonomía de los coordinadores de las unidades fueron consideradas como un aspecto positivo de ese nuevo modelo en la visión de los entrevistados.

La creación de unidades sectoriales mejoró, yo creo que quedó mucho más fácil. Un hospital como el [nombre del hospital] es muy grande para tener solo una coordinación, sólo una gerente, para que ella sepa todo del hospital, saber de todas las áreas del hospital, yo creo que es inviable. Con las coordinadoras, con la autonomía que les es dada, yo creo que es más fácil desarrollar un trabajo mejor. (E07).

Veo el trabajo de coordinación como un trabajo de apoyo, yo estoy viviendo eso y lo veo bastante positivo. Se delega más autonomía a las unidades, uno siente más autonomía para decidir. Con esa descentralización fui más rápido (E09).

Los cambios trajeron algunas dificultades a aquellos profesionales que asumían funciones gerenciales, independientemente del nivel jerárquico. Mintzberg ${ }^{30}$ resalta que las nuevas formas de gestión traen a trabajadores de la mediana administración la presión por la productividad y por la eficiencia, así como la preocupación con la posibilidad de despido, caso el trabajador no se adapte al nuevo modelo. Los entrevistados mencionaron la preocupación con resultados, con facturación, con reducción de costos y con el riesgo de despido, conforme puede ser constatado en los relatos a continuación.

La mayor dificultad es que nos quedamos muy preocupadas con la parte administrativa también, con lo que está siendo hecho, lo que va a dar y lo que no va a dar resultado al final, a la hora de facturar. Hoy día es completamente diferente. El cobro es mucho mayor. Ud. es responsable por el resultado final de su sector (E01).

Ella [coordinadora] nos trae mucho la cuestión de los costos mensuales, así, este mes estamos gastando mucho en esto, vamos a pensar si hay necesidad de gastar todo eso. (E04).

Quién no se adecuaba al perfil [...] mucha gente se fue. (E09)

Una dificultad [para la coordinadora] es que los sectores no son integrados, están esparcidos por el hospital (E06).

La percepción de los entrevistados en cuanto a la nueva forma de gestión del hospital denota una significativa alteración en las prácticas de trabajo de la organización, remitiendo a la necesidad de profundización sobre el día a día de esos profesionales, aspecto que ocupa un lugar privilegiado en la configuración de la identidad. ${ }^{3,4}$

Por tanto, se buscó conocer el cotidiano de trabajo de los enfermeros supervisores, es decir, los aspectos relacionados con el día a día del enfermero en la función gerencial. Los enfermeros supervisores entrevistados fueron solicitados para que describan las facilidades y las dificultades encontradas en el ejercicio de sus funciones, así como, los aportes que estos creen traer a la organización. Inicialmente, como puntos facilitadores para la realización de las actividades gerenciales se resaltaron aspectos relacionados con la relación interpersonal, tales como, motivación y valoración, apertura para exponer la opinión y mejor comunicación, confirmando componentes del proceso de identificación del sujeto como sentimiento cognitivo, afectivo y valorativo con relación al hecho de pertenecer a determinado grupo social. ${ }^{18}$ 
Al ser indagados sobre los puntos dificultadores enfrentados en sus rutinas, los enfermeros supervisores destacaron: la burocratización, el retrabajo en corregir errores, el cuadro de personal disminuido, además de la dificultad para administrar sectores físicamente distantes.

Sobre los aportes que estos traen a la institución al efectivizar sus funciones, se resalta la satisfacción del cliente, como producto final de sus acciones. Además, tres entrevistados entienden que el profesional contribuyó mucho con la institución, al facturar correctamente.

Sus respuestas corroboran la premisa de Avelar $^{43}$ sobre la necesidad de relaciones jerárquicas más horizontalizadas y la realización de reuniones más frecuentes, no solo de índole administrativao, sino también científica y de integración. De esta forma, el día a día de los enfermeros supervisores estaría amenizado con el equilibrio en sus actividades, así como con la posibilidad de que estos obtengan más satisfacción en sus roles.

\title{
La formación en enfermería y la práctica profesional
}

Al ser preguntados sobre la articulación entre formación en enfermería y actuación profesional del enfermero en la función gerencial, la mayoría de los enfermeros supervisores entrevistados afirmó que existe una disparidad entre formación y práctica, factor que influye directamente en la actuación del gerente, conforme muestran los relatos a continuación.

\begin{abstract}
Nos formamos totalmente enfocados hacia la asistencia. No tenemos noción, salimos sin tener noción sobre las cuestiones administrativas que vamos a enfrentar el día a día (E01).

Yo creo que la persona no está muy bien preparada, por lo menos en la época que yo estudié, no había énfasis en la cuestión. Entonces con relación a lo que estudié, yo creo que el enfermero precisa hacer otros cursos, él precisa ir a la administración, gerenciamiento propiamente dicho. No es suficiente, creo que no. (E02).

Yo creo que sí influye, porque esa parte administrativa en la facultad todavía es muy superficial. Entonces cuando llegamos, prestamos mucha atención a la asistencia. Pero, por detrás de una buena asistencia, una buena técnica, Ud. tiene que saber administrar. Yo creo que el error también es nuestro. Pensamos "ah, voy a llegar al hospital y voy a dar asistencia”. Pero, ¿y la parte del administrativo? La cuestión de estar reasignando empleados, calculando, la cuestión del abastecimiento de material, entonces uno hace todo a un lado, cree que no va a trabajar con eso, pero todo el tiempo estamos haciendo eso. Yo creo que la deficiencia empieza en la facultad, tanto de parte de los profesores, para llamarnos la atención hacia eso, como también por parte del alumno, y así queda. Solamente cuando uno entra al mercado es cuando se enfrenta con ello. (E05).
\end{abstract}

La disonancia entre la formación y la práctica profesional en las organizaciones planteada por los enfermeros supervisores entrevistados, y observada en otros estudios ${ }^{5,7}$, interfiere en el proceso de construcción de identidad. Dubar ${ }^{4}$ entiende que la primera identidad profesional "tiene cada vez más oportunidades de no ser definitiva. Ella es regularmente confrontada con las transformaciones tecnológicas, organizacionales y de gestión del empleo, estando destinada a sufrir ajustes y cambios sucesivos"4:150. La perspectiva del autor mencionado es reafirmada en este estudio, así como, en otros realizados en hospitales de Belo Horizonte. 1,9 $^{1}$

A pesar de haber sido considerada deficiente, en la percepción de los entrevistados, la formación gerencial en la graduación no impidió que ellos asumieran cargos gerenciales. La calificación gerencial forma parte de su realidad en el actual contexto, como puede ser constatado, a continuación, en los relatos sobre los requisitos de un enfermero ideal para la función gerencial. 


\title{
Requisitos de un enfermero ideal para la función gerencial
}

Bajo la percepción de los entrevistados, los requisitos que se destacan para ser un gerente enfermero ideal son aquellos relacionados con el dominio de la profesión, tanto en el ámbito gerencial como asistencial, confirmando la tendencia identificada en estudios anteriores sobre las actividades del profesional de enfermería en hospitales privados. ${ }^{1,8,9}$ Además, algunos entrevistados entienden que el gerente debe tener la habilidad de comunicación y la capacidad para estimular e involucrar a los empleados con el trabajo. Las exposiciones a continuación presentan los requisitos de un enfermero ideal para la función gerencial, mencionados por entrevistados.

\begin{abstract}
Enfermera gerente es la enfermera que conoce mucho nuestra teoría asistencial. Creo que tiene que saber, porque todo el grupo precisa la técnica. Gerencial, la persona tiene que tener una visión amplia, no sólo asistencial, sino del negocio, porque hoy día la tendencia es esa. Si Ud. es gerente de un sector, su sector es una microempresa. Él tiene que dar resultado (E01).
\end{abstract}

Él tiene que entender acerca de procesos, tiene que entender sobre protocolos del servicio, él tiene que comprometerse tanto en la parte administrativa como en la parte asistencial. Yo creo que él tiene que saber comunicar (E07).

Es aquella persona que nos estimula, que motiva a las personas, que tenga una capacidad muy grande de percepción y que perciba lo que influye a las personas en el momento en que ellas no están motivadas; lo que está interfiriendo en la capacidad de desarrollar el trabajo (E03).

Los enfermeros supervisores entrevistados mencionan también la importancia de asumir su nuevo rol, incluso como referencia para el equipo, cuando nuevas formas de gestión son incorporadas en su práctica. Por tanto, ellos reproducen el discurso de la organización.

\begin{abstract}
Cualquier cargo directivo se torna espejo de sus empleados. Yo creo que uno tiene que tomar mucho cuidado con la parte del comportamiento. Puntualidad, responsabilidad, compromiso, tiene que querer tener visión del todo, visión del negocio, de la administración. Porque gerenciar no es coordinar un equipo de enfermería. Si Ud. no muestra resultado financiero a fin de mes, quién no trabajó fue Ud. El rol del enfermero cambia. Él no es un enfermero gerencial asistencial; él es un enfermero gerencial administrativo. Él administra personal, administra costos y todo el resto (E01).
\end{abstract}

Los requisitos expuestos remiten hacia la socialización secundaria ${ }^{28}$, es decir, a la identificación ${ }^{13,23}$ de los enfermeros supervisores con la organización donde actúan. Goffman $^{26}$ (1959) destaca que la capacidad del individuo para impresionar al otro es una actividad significativa en la constitución del yo (identidad), así como, demuestra la influencia recíproca de los individuos sobre las acciones unos de otros, cuando en presencia física inmediata. El mismo autor resalta que en esa interacción social el individuo representa roles, preestableciendo un patrón de acción que será desarrollado durante la representación, confirmando la mención de Dubar ${ }^{3,4}$ sobre la importancia del mundo laboral y su significado en la construcción de la identidad.

Teniendo en cuenta que la identidad profesional de base no es $\mathrm{fija}^{3,4}$ y que el contexto en el cual las organizaciones hospitalarias están insertas ha sido marcado por expresivos impulsos para transformaciones ${ }^{35,36}$, se buscó conocer los planes profesionales de los enfermeros supervisores, teniendo en cuenta que la proyección del futuro es componente de su identidad. 


\section{Expectativas de carrera de los enfermeros supervisores}

En cuanto a los planes profesionales, con la intención de conocer la proyección de futuro de esos profesionales enfermeros supervisores, se observó una cierta disonancia entre la formación del enfermero y su práctica profesional. ${ }^{5-7}$ Su deseo manifiesto más presente fue el de continuar estudiando. Los planteamientos de los entrevistados estaban marcados por la voluntad de buscar un perfeccionamiento a nivel de especialización, maestría e, incluso, doctorado.

\footnotetext{
Ya está pasando la hora de hacer un posgrado, especialización. Después pretendo hacer una maestría. (E02)

Continuar estudiando, siempre, invirtiendo en mí, a pesar de que hace diez años [de formada], yo digo que no sé nada. Porque las cosas están cambiando. La postura de la enfermera está cambiando, con ello uno tiene que cambiar la postura (E03)

Voy a concluir mi curso de especialización en auditoría, más adelante voy a hacer una maestría, un doctorado, porque quiero dar secuencia en eso. Porque me parece que la enfermería aun está muy deficiente. Hasta aquí está bien, pero para mí no es suficiente. Estoy molesta. Preciso algo más. (E04).
}

Además de la necesidad de reducir el gap apuntado teóricamente, las exposiciones denotan también un interés en continuar estudiando, independientemente de cuestiones relacionadas con la formación asistencial y gerencial. Se trata también de demandas del actual contexto de la "modernidad líquida", en el cual las personas viven en la búsqueda continua de conocimiento $^{44}$, como forma, incluso, de no ser excluídas. ${ }^{20}$

Los entrevistados mencionaron también el deseo de, futuramente, trabajar en otra área como dermatología, consultoría, laboratorio y atención médica de emergencia. Se observa la expectativa de los entrevistados en cambiar el área de actuación, es decir, buscar nuevas formas de trabajo, nuevos grupos sociales. Esto puede ser el resultado de una comparación ${ }^{18}$, ya que, los grupos citados, particularmente, la emergencia, se están destacando socialmente, conforme lo demuestran estudios en el área de administración sobre la identidad de los enfermeros de un servicio de atención móvil de urgencia (SAMU). ${ }^{43}$ Conforme el estudio de Avelar ${ }^{43}$ los enfermeros que trabajan en emergencias, en especial en el SAMU, se sienten portadores de un gran poder y poseedores de dominio de la técnica y del cuidado y conocimiento global de las acciones de salud. Además de la preparación técnico-científica y emocional, los enfermeros hacen referencia a sí mismos y a sus colegas de trabajo como personas vanidosas, elitistas.

El proceso de reconocimiento recíproco de estos sujetos depende de la legitimación de la identidad asociada a los saberes, competencias e imágenes de sí propuestas y expresadas por los indivíduos. ${ }^{43}$ Paralelamente, Abreu $^{45}$ justifica que la transición por la cual la enfermería viene transitando implica que sus agentes tienden a acompañar patrones de formación cada vez más elevados y diferenciados. Esta búsqueda de reconocimiento es justificada por Dubar ${ }^{3}$ como una forma de afirmar la identidad para sí por medio de la mirada del otro.

El interés de los entrevistados de pertenecer a grupos positivamente distintos señala una identidad social no satisfactoria. Tajfel y Turner $^{18}$ afirman que los individuos se esfuerzan por conseguir o mantener una identidad social positiva. Según los autores, una identidad social positiva está basada en comparaciones favorables que pueden ser hechas entre los miembros del grupo y aspectos relevantes de los grupos externos: el grupo debe ser percibido como positivamente diferenciado o distinto de los aspectos externos del grupo. 
Cuando esa identidad social no es satisfactoria, los individuos se esfuerzan para dejar su grupo ya existente y juntarse a algún grupo más positivamente distinto ${ }^{18}$, siendo el rescate de emergencia un ejemplo emblemático de un grupo positivamente distinto. ${ }^{43}$ Uno de los entrevistados menciona explícitamente el deseo de éxito y reconocimiento profesional.

Se resalta que una enfermera supervisora respondió la cuestión sobre planes profesionales con la afirmativa de que desea ser madre. Solamente esta profesional afirmó también que tiene planes de asumir la coordinación, o sea, la función gerencial jerárquicamente superior. La manifestación del deseo de realización personal, como madre y, profesional, como gerente, es un típico conflicto de identidad relacionado con cuestiones de género, conforme identificó Brito ${ }^{1,8,9}$.

Entre los planes profesionales, una posibilidad vislumbrada para los entrevistados sería un cargo gerencial, teniendo en cuenta que los enfermeros han expandido sus actividades y ocupado ese espacio de trabajo. ${ }^{1,9}$ Al ser cuestionados directamente sobre el interés por asumir la gerencia, caso fueran invitados, parte de los enfermeros supervisores respondieron que no. Las justificativas presentadas fueron: inmadurez, falta de flexibilidad y de habilidad política y falta de preparación en el ámbito de la formación gerencial. Se destaca que no ha sido descartada por los entrevistados la posibilidad de asumir el cargo gerencial en el futuro, conforme sus relatos a continuación

\footnotetext{
Por ahora, no. Por varios motivos. Creo que soy muy inmadura. Creo que tengo que trabajar acerca de mis relaciones. Todavía estoy en aquella fase de que lo correcto es eso y así tiene que ser. Entonces tengo que trabajar ese lado de la flexibilidad, la enfermera tiene que ser flexible, tiene que ser política. Me parece que tengo que trabajar mucho ese aspecto. (E08).
}

Yo creo que no me siento preparada para asumir esa función aquí dentro de la institución. Me parece que todavía no es el momento. No me sentiría a gusto para estar asumiendo eso ahora. Es tan complejo. Creo que todavía me falta un curso de administración. (E07)

Por ahora no. Tal vez en el futuro, tal vez en otro momento. (E02).

Ah no, gerencial, no. No, no aceptaría. Es muy reciente, creo que me falta experiencia, porque es un desafío muy grande. Lógico que es tentador, pero me parece que por ahora no. En un futuro, ¿quién sabe? Pero creo que precisaría más experiencia. (E06).

Otro análisis, conforme Brito et al. ${ }^{1}$, se refiere al hecho que la preparación está vinculada a la experiencia y al tiempo de servicio en las instituciones. El tiempo de trabajo en la organización o en el propio sector en que los enfermeros actúan es señalado como un factor que acredita al enfermero para la realización de sus actividades, otorgándole una mayor autonomía y poder de decisión. La situación descrita está vinculada al conocimiento técnico de las gerentes que, asociado a los aspectos conceptuales y relacionales, se consolida como una competencia fundamental para el ejercicio de la gerencia y, por tanto, para la reconfiguración de su identidad profesional.

\section{CONSIDERACIONES FINALES}

El presente estudio fue realizado con el propósito de analizar la configuración de identidad de enfermeros supervisores de un hospital privado de gran tamaño ubicado en Belo Horizonte. Para su concretización, se utilizó la metodología descriptivo analítica, de abordaje cualitativo, en la modalidad de estudio de caso. Mediante el análisis de los datos, se evidenció que el hospital, escenario del estudio, pasaba por intensas transformaciones 
organizacionales, siendo un espacio fundamental en la construcción de la identidad del enfermero supervisor. Tal evidencia está vinculada al hecho que las organizaciones hospitalarias están marcadas por relaciones sociales, vividas de diversas formas por los actores involucrados en el ejercicio de sus funciones.

La percepción de los enfermeros supervisores en cuanto a la nueva forma de gestión del hospital donde actuaban, denota una importante ruptura en su trayectoria de identidad, cuando el modelo de gestión tradicional fue reemplazado por un nuevo modelo, con la finalidad de sostener la adaptación al contexto competitivo y a la supervivencia de la organización en el mercado.

El día a día de trabajo del enfermero supervisor se caracterizó por el abordaje de puntos facilitadores para la realización de las actividades gerenciales, así como, sus atribuciones a la institución por medio de sus funciones. Se observaron aspectos de la relación interpersonal, tales como, motivación, valoración, apertura para plantear opiniones y una mejor comunicación.

En cuanto a la formación en enfermería y actuación profesional del enfermero en la función gerencial, verificándose la articulación entre la academia y el ejercicio laboral, se constató que la mayoría de los enfermeros supervisores entrevistados afirmó la existencia de disparidad entre formación académica y práctica profesional, factor este que influye directamente en la actuación del enfermero, así como, en su constitución de identidad.

La burocratización, el retrabajo en corregir errores, el cuadro de personal disminuido, además de la dificultad para administrar sectores físicamente distantes fueron mencionados como factores dificultadores para la actuación del enfermero supervisor, estando vinculados al momento de transición y reestructuración de la institución. Sobre las contribuciones que estos traen a la institución al efectivizar sus funciones, se resalta la satisfacción del cliente y acciones vinculadas al correcto funcionamiento.

En la percepción por parte de la mayoría de los entrevistados, los requisitos que se destacan para ser un gerente ideal son aquellos relacionados con el dominio de la profesión tanto en el ámbito gerencial como en el asistencial, habilidad de comunicación y la capacidad para estimular y comprometer a los empleados con el trabajo.

Los enfermeros supervisores al ser abordados sobre sus planes profesionales, con la intención de conocer la proyección de futuro de esos profesionales, fueron categóricos al manifestar el deseo de continuar estudiando. Las exposiciones de los entrevistados fueron marcadas por la voluntad de buscar el perfeccionamiento a nivel de especialización, maestría y doctorado.

Ante los resultados obtenidos en este estudio, se puede reafirmar que las identidades sociales y profesionales son resultados de construcciones sociales basadas en las trayectorias individuales, en los procesos de formación y en las relaciones de trabajo. Reflejan, por tanto, configuraciones de identidad específicas de situaciones históricas y de momentos productivos de la sociedad. De esa forma, se destaca la identidad como en constante cambio. En este estudio, fue evidenciado cómo enfermeros supervisores que actúan en un hospital de gran tamaño, ubicado en Belo Horizonte, construyen su identidad, siendo relevante y enriquecedora la realización de nuevos estudios que puedan llegar a completar o ampliar las bases aquí construidas. 


\section{REFERENCIAS}

1. Brito MJM, Lara MO, Soares EG, Alves M, Melo MCOL. Traços identitários da enfermeira-gerente em hospitais privados de Belo Horizonte, Brasil. Saude Soc. 2008; 17(2):45-57.

2. Barros VA, Nogueira MLM. Identidade e trabalho: reflexões a partir de contextos precarizados e excludentes. Rev Educ Tecnol. 2007 set/dez; 12(3):10-12.

3. Dubar C. A socialização: construção de identidades sociais e profissionais. $2^{\mathrm{a}}$ ed. Portugal: Editora Porto; 1997. 239p.

4. Dubar C. A socialização: construção de identidades sociais e profissionais. São Paulo: Martins Fontes; 2005. 343p.

5. Gabrielli JMW. Formação do enfermeiro: buracos negros e pontos de luz [tese]. São Paulo: Escola de Enfermagem da Universidade de São Paulo; 2004.

6. Brasil. Ministério da Educação. Secretaria de Educação Superior. Diretrizes Curriculares para os Cursos de Graduação. Resolução CNE/CES № 3, de 7 de novembro de 2001. [Acesso em 2009 nov. 10]. Disponível em: http://www.mec.gov.br/sesu/diretriz.htm.

7. Peres AM, Ciampone MHT. Gerência e competências gerais do enfermeiro. Texto Contexto Enferm. 2006 sept; 15(3). [Citado em 2009 nov. 10]. Disponivel em: http://www.scielo.br/scielo.php?script=sci_arttext\&pid $\quad=$ S0104-7072006000300015\&ln $\mathrm{g}=\mathrm{en} \& \mathrm{nrm}=\mathrm{iso}$.

8. Brito MJM, Monteiro PRR, Costa JO. Interfaces da mudança e dos modos de gestão na rede hospitalar privada de Belo Horizonte: caminhos e descaminhos na ótica da gerenteenfermeira. In: ENANPAD, 26, 2002, Salvador. Anais... Salvador: ANPAD; 2002.

9. Brito MJM. A configuração identitária da enfermeira no contexto das práticas de gestão em hospitais privados em Belo Horizonte. [tese]. Belo Horizonte: Faculdade de Ciências Econômicas, Universidade Federal de Minas Gerais; 2004.

10. Roquete FR. Impactos da regulamentação estatal na gestão organizacional: o caso de uma cooperativa médica Unimed no Estado de Minas Gerais [dissertação]. Belo Horizonte: Centro de Pós-Graduação e Pesquisas em Administração da Faculdade de Ciências Econômicas da Universidade Federal de Minas Gerais; 2002.

11. Pompeu JCB. A situação contratual da rede hospitalar privada vinculada ao SUS: alguns elementos para reflexão [dissertação]. Rio de Janeiro: Escola Nacional de Saúde Pública da Fundação Oswaldo Cruz/MS; 2004.

12. Machado HV. A Identidade e o contexto organizacional: perspectivas de análise. RAC. 2003; (Ed Especial):51-73.

13. Fernandes MER. Os paradoxos do processo identificatório na trajetória de diretores, gerentes e técnicos de duas empresas multinacionais de tecnologia. In: Anais do Encontro Nacional da Associação Nacional dos Programas de Pósgraduação em Administração, 33, 2009, São Paulo.São Paulo: ANPAD; 2009.

14. Freud S. A experiência de satisfação. In: Freud S. Publicações pré-psicanalíticas e esboços inéditos. 2. ed. Rio de Janeiro: Imago; 1987. Parte I, cap. 11, p.336-7. Obras Psicológicas Completas. Edição Standard Brasileira.

15. Erikson EH. Identidade: juventude e crise. Rio de Janeiro: Guanabara; 1987.

16. Castells M. O poder da identidade. $2^{2}$ ed. São Paulo: Paz e Terra; 1999. v. 2. 530p.

17. Tajfel H. Social psycology of intergroup relations. Ann Rev Psychol. 1982; 33:1-39.

18. Tajfel H, Turner JC. An integrative theory of intergroup conflict. In: Hatch MJ, Schultz M, editors. Organizational identity. Oxford: Oxford University Press; 2004.

19. Hall S. A identidade cultural na pós-modernidade. In: Hall DH, McGrew T. Modernity and is futures. $10^{\mathrm{a}}$ ed. Rio de Janeiro: DP\&A Editora; 2005. $102 \mathrm{p}$.

20. Bauman Z. Identidade: entrevista a Benedito Vecchi/Zygmunt Bauman. Rio de Janeiro: Jorge Zahar; 2005. 110p.

21. Bauman Z. Vida líquida. $2^{a}$ ed. Rio de Janeiro: Jorge Zahar; 2007. 212p.

22. Ésther ABA. Construção da identidade gerencial dos gestores da alta administração das 
universidades federais de minas gerais. 2007 [tese]. Belo Horizonte: Universidade Federal de Minas Gerais; 2007. [Citado em 2009 ago. 28]. Disponível em: http://www.cepead.face.ufmg.br/index.php?option=com_wrapper\&view=wrapper\&ltemid=209 23. Fernandes MER. De "Jóia da Coroa" a "Coroa sem Jóia": estudo do processo identificatório em duas empresas multinacionais em reestruturação [tese]. Belo Horizonte: UFMG; 2008.

24. Cooley $\mathrm{CH}$. Society and the individual. In: Hatch MJ, Schultz M, editors. Organizational identity: a reader. New York: Oxford University Press; 2004.

25. Mead GH. Mind, self and society: from the Standpoint of a Social Behaviorist. Chicago: University of Chicago Press; 1934. p.113-9.

26. Goofman E. The presentation of self in everyday life. New York: Doubleday; 1959.

27. Goofman E. Estigma: notas sobre a manipulação da identidade deteriorada. $4^{\underline{a}}$ ed. Rio de Janeiro: LTC; 1988.

28. Berger PI, Luckmann T. A construção social da realidade. 3aㅡ ed. Petrópolis: Vozes; 1976. 29. Chanlat JF. Ciências sociais e management: reconciliando o econômico e o social. São Paulo: Atlas; 2000.

30. Mintzberg H. Produtividade que mata. GVExecutivo. 2007 nov/dez; 6(6):17-23.

31. Cecílio LO. Mudar modelos de gestão para mudar o hospital: cadeia de apostas e engenharia de consensos. Rev Espaço para a Saúde. 2000 jun; 1(2):04-26.

32. Bernardino E, Felli VEA. Saberes e poderes necessários à reconstrução da enfermagem frente a mudanças gerenciais num hospital de ensino. Rev Latino-Am Enferm. 2008; 16(6). [Citado em 2009 out. 20]. Disponível em: http://www.scielo.br/scielo.php?script=sci_arttext\&pid=S0104$11692008000600015 \&$ lng $=$ pt\&nrm=iso.

33. Gonçalves JEL. Os novos desafios da empresa do futuro. RAE. 1997 jul/set; 37(3):10-9. 34. Castro JHM, Kilimnik ZM, Sant'Anna AS. Modernidade organizacional em gestão de pessoas como base para a incorporação de modelo de gestão por competências RAC Eletron. 2008 jan/abr; 2(1):105-22.

35. Organização Nacional de Acreditação-ONA. [Citado em 2009 out. 12]. Disponível em: http://www.ona.org.br/site/internal_institucional.jsp?pagesite=oqe.

36. Brasil. Ministério da Saúde. Secretaria de Assistência à Saúde. Manual Brasileiro de Acreditação Hospitalar. Secretaria de Assistência à Saúde. 3aㅡ ed. Brasília: Ministério da Saúde; 2002.

37. Chizzotti A. A pesquisa em ciências humanas e sociais. São Paulo: Cortez; 1991. 38.Vergara SC. Projetos e relatórios de pesquisa em administração. $2^{a}$ ed. São Paulo: Atlas; 1998.

39. Yin RK. Estudo de caso: planejamento e métodos. $2^{\mathrm{a}}$ ed. Porto Alegre: Bookman; 2001. 40. Minayo MCS. O desafio do conhecimento: pesquisa qualitativa em saúde. $7^{a}$ ed. São Paulo: Hucitec; 2000.

41. Bardin L. Análise de conteúdo. Lisboa: Edições 70; 1977.

42. Mintzberg H. Burocracia Profissional. In: Mintzberg H. Criando organizações eficazes. $2^{a}$ São Paulo: Atlas; 2003. p. 212-38.

43. Avelar VLLM. Configurações Identitárias de enfermeiros de um serviço de atendimento móvel de urgência [tese]. Belo Horizonte: Faculdade Novos Horizontes; 2009.

44. Castells M. A sociedade em rede. 8르 ed. São Paulo: Paz e Terra; 2005.

45. Abreu WC. Identidade, formação e trabalho: das culturas locais às estratégias identitárias dos enfermeiros. Estudo Multicasos. Lisboa: Educa; 2001. 
๑ COPYRIGHT Servicio de Publicaciones - Universidad de Murcia 